\title{
System-targeted approach to the integrated use of transport in the interests of life safety
}

\author{
Ruben Kazaryan ${ }^{1, *}$ \\ ${ }^{1}$ Moscow State University of Civil Engineering, Yaroslavskoe shosse, 26, Moscow, 129337, Russia
}

\begin{abstract}
The technical component of the MTE system (man-technologyenvironment), a special case of which are the means of mechanization and transportation (MMT) in construction, is investigated as it develops in terms of reliability and efficiency of the processes of construction production implemented using MMT. Firstly, there is no evaluation of the economic justification for an integrated transport communication system. Secondly, there are economic and mathematical models of the integrated use of different types of transport in the interests of ensuring environmental safety. Thirdly, there are methodologies of economic justification of the complex use of transport in the interests of environmental safety. Certification of means of mechanization of manual labor and transportation of building cargo and structures (MMT) is one of the important packages of construction production objects for services of high-rise construction. The variety of certification aspects corresponds to the variety of domestic and foreign production used in the conditions of market competition in the construction of MMT, in particular, the means of road transport in construction (MRTC). High dynamics of transport support for economic, social, demographic, and environmental security in a market economy require the creation of a concept for the integrated use of transport in the interests of Russia's national security. The demand for systematic consideration of three components (human, engineering, and environment) from the positions of system engineering of construction, the theory of functional systems and modeling of their mutual influence is identified with the main role of the subject-object approach to the comfort and workability criteria, organizationaltechnological and organizational-anthropotechnical reliability of the MTE system.
\end{abstract}

\section{Introduction}

The development of construction production is not only an engine, but also an indicator of the investment climate and the level of the country's economic development. Strict competition in this segment of the market makes us constantly improve equipment and technologies. High rates of development and comprehensive construction are provided not only by the actual construction services, but also by packages of construction services. Works in construction are carried out with the help of mechanization of manual labor and transportation of construction cargo and structures. Certification of means of mechanization

\footnotetext{
${ }^{*}$ Corresponding author: r.kazarian@mail.ru
} 
of manual labor and transportation of building cargo and structures (MMT) is one of the important packages of construction services. The variety of aspects of certification corresponds to the diversity used in the conditions of market competition in the construction of MMT of domestic and foreign production, in particular - road transport in construction (MRTC). Important parts of MRTC, which significantly affect the level of quality of their operation and the performance of construction and installation works, include the driver's cab and its internal environment (mobile workplace of construction production) [1-5].

These new organizational forms of the development of OTR (organizational and technological reliability) proved to be much more complicated than purely technical systems, since they contain human interaction with the external environment. This interaction has a probabilistic character specific for each individual (individually). In this regard, there is a personal norm of interaction with the external environment and adaptation to it for each person (the adaptive norm of a particular person). The study of the specifics of the formation of the adaptive norm of a person, its manifestation, and compensation of the pathologies that arise while ensuring the safety of life and productive activity of man is one of the most important tasks of science in a democratic society [4-9].

\section{Methods}

The possibilities of different methods, techniques, and instruments for evaluation and diagnostics of various processes and manifestations of the human body have been studied with sufficient adequacy, reliability, and accuracy to record the response of a particular person to anthropogenic, technogenic, and environmental effects of varying intensity and duration. The gas-discharge visualization method (GDV-method) with the properties of efficiency, sufficient accuracy and repeatability of the measurement values, the ability to visualize the results of diagnostics and to form on their basis a generalized visually perceptible image of the health resource and the dynamics of its change is chosen as the most adequate to the tasks of the research.

The increase in OTR in optimizing the servicing subsystems of the construction industry is achieved in two ways: by decreasing the level of influence of the factors affecting the failure of the reliability of the functioning of these subsystems (such a reduction is not always possible due to the complexity and often impossibility of influencing these factors) and the creation of subsystems sufficiently reliable functioning under the influence of negative factors. The increase in the MTE of the servicing subsystems of construction production is affected by a decrease in the level of influence of pathogenic factors by combining these two directions, as well as by improving the "operator-workplace" system. In the comparative analysis of the quality and comfort of closed mobile workplaces (CMW) for cabins of various modifications of vehicles for mechanization of construction production, the methodology and automated technology of rapid quality assurance of CMW, developed by the Research Laboratory "Information Technologies, Economics and Complex Security" (ITECS) of the Moscow Chamber of Certification in Transport for monitoring the parameters of the "manCMW-activity" system in the construction industry, as well as for implementation of theoretical and applied research in the field of territorial and topological analysis, risk management, and compensation of pathological effects in the system "man-technologyenvironment" (MTE), have been introduced and passed trial operation at the enterprises of Automobile plant "Ural" and Uralspetstehnika. The results of numerous studies by the ITECS laboratory allows using personal computers and engineering facilities of gas-discharge imaging (GDV cameras) to take scientifically grounded decisions on the formation of qualitative systems "man-CMW-activity" in which the driver or machinist of the means of mechanization of construction work can comfortably carry out construction production activities, and also provide the opportunity to reduce the resource costs of designers of CAD 
systems for construction, to improve the quality, and to reduce the development of projects for new and modernized means for mechanization of construction work [4-10].

Harmonization of the device, functioning of the device, the totality of the solved tasks or activities are applied to the MTE system - mutual agreement, establishment of equability, harmony, balance between problems and tasks of a similar priority level (equally requiring implementation in a given period); coordination, ordering, ensuring mutual conformity of relation processes in each of the components of the MTE system and in the system as a whole. Harmonization is carried out by a consciously managed distribution between the components of the MTE system of limited resources by priority. Priorities are the criteria that influence the definition of the relative importance of problems and tasks, and the elimination of existing "conflicts of interest" caused by limited resources. As far as we know, there are three directions for the development of the theory of functional systems [1-6, 8-16,19-26].

The first direction is general philosophical and methodological research, which establishes a "conceptual bridge" between the concept of "functional system" (as a scientific and philosophical category) and its application to any studied object (P.K. Anokhin, K.V. Sudakov, their disciples and followers). The task of this direction is to make the theory of functional systems sufficiently formalized and applicable for the purpose of improving the cognitive process in the pre-conceived areas by systematization and generalization of empirical data, i.e. to turn it into an instrument of applied scientific research.

The second direction is the study of functional systems that actually exist in biology, physiology, physics, medicine, technology, sociology, economics and other scientific and practical areas of human functioning and activity. The listing of numerous eminent researchers in this area within the framework of the paper seems inexpedient. The task of this direction is to overcome (with the help of the first direction of development of the theory of functional systems) a narrow analytical approach in research work and to lay a "conceptual bridge" between synthetic and analytical approaches in research.

The third direction is the mathematical formalization of the concept of a functional system as an organic component of the mathematical theory of systems. Until recently, this direction declared an unshakable principle: they formulate a mathematical model on a purely theoretical basis, and only then proceed from it to explain the real properties and manifestations of any (biological, physical, technical, social, industrial, economic, etc.) of the system, including the functional one, i.e. the theoretical mathematical modeling must precede the applied research.

However, there is also an opposing view: true systemic patterns can be developed only as a result of applied research in biology, physics, engineering, and also in the study of "large" socio-economic and production systems. According to the founders of the national system movement (P.K. Anokhin, K.V. Sudakov, etc.), this material should be the basis of mathematical analysis and strict formalization of system regularities. From the earliest times, the system is regarded as one of the most important characteristics of scientific knowledge. Its ideas were expressed even by the ancient Greeks and Romans, who performed the analogy of a significant amount of empirical material, but never rose to philosophical generalizations of the results. Stygian and unconscious (at the level of "common sense"), the use of elements of systemic nature-knowledge for many centuries served to study the final results of goalsetting, in relation to which it was necessary to perform system description and classification [1-6, 8-16,19-27].

\section{Results}

As studies have shown, the following triad is less vulnerable: source data - specially presented initial data - estimates under current conditions. The latter scheme does not provide for an annalistic idealization in a general form, but has only a description of each of the units 
of input data, consistent with the requirements of a particular task. At the same time, the simplification of real regularities is eliminated for any attempt to approximate them using relatively simple means. Mathematical statistics, as a rule, provides for this kind of idealization of distribution, which simplifies its use, but the quality of the subsequently obtained estimates is by no means flawless. However, when solving practical problems, it becomes necessary to use criteria of an arbitrary kind. In particular, in the case when it is necessary to give the accuracy indices of numerical solutions with respect to given observations, only approximately representing the true values, and also in the case when the accuracy of solutions is not lower than the predetermined one, it is reasonable to base on the so-called threshold criterion, rather inconvenient from the point of view of analytical calculations. The paper uses such procedures for approximating hidden regularities, which can be attributed to the type of completely formalized procedures (analytical formalization is meant, i.e. giving a certain form - algorithmic, tabular, matrix, topological, etc.). They are characterized by the order of information processing shown in Fig. 1:

- the initial sample in a one-time order is used to find the approximating expression common to all conditions, after which the information contained in it can be forgotten, since its further application is not foreseen; - estimates for any conditions are found by substituting the current values of factors into the same approximating expression formed in the previous step.

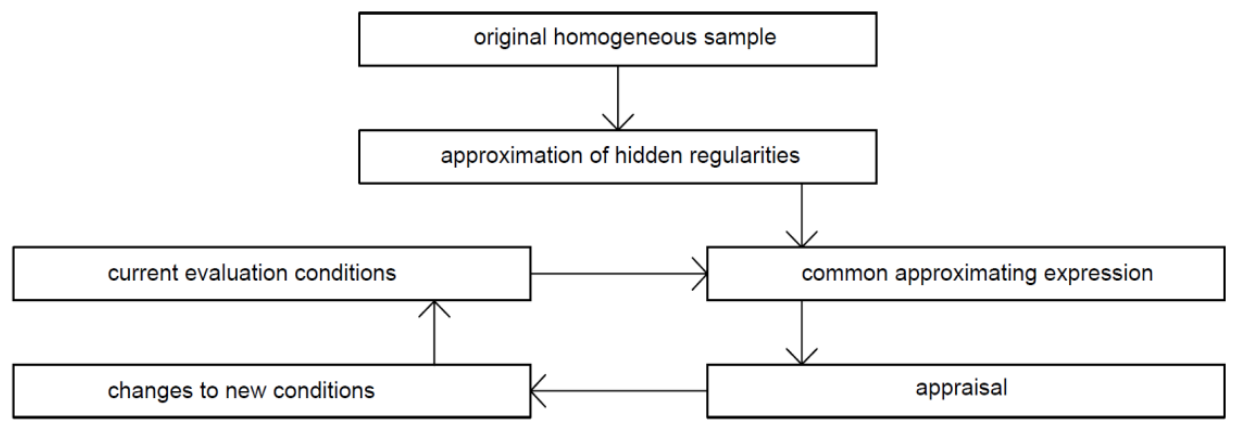

Fig. 1. The structure of evaluation when used analytically formalized procedure for approximating hidden regularities.

Thus, when formalized procedures are implemented, all points of the state space of the MTE system are assigned the same laws; consequently, these procedures assume homogeneity of the initial sample. In the case of a heterogeneous initial sample, the use of fully analytically formalized procedures cannot be considered correct because the variability of the regularities from point to point in the observation space cannot be estimated on the basis of a uniform approximating expression for all conditions. In the case of a non-uniform sample, the analytical formalization of the general approximating expression is excluded. Elements of such formalization can be used only in the stage of obtaining estimates for particular conditions. Thus, a procedure is introduced with incomplete analytical formalization or a semi-formalized procedure (Fig. 2), which ensures the correctness of the solution of estimation problems in the case of an inhomogeneous sample. 


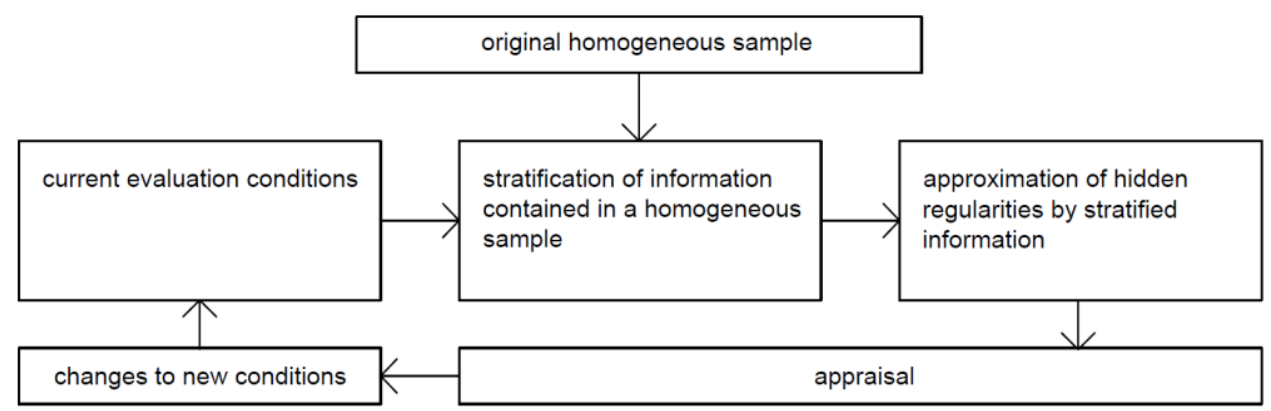

Fig. 2. The structure of evaluation when used analytically semi-formalized procedure for approximating hidden regularities.

Only the accuracy indicators of the estimation are a fairly large set of possibilities. For clarity, their classification is shown in the form of a scheme in Fig. 3. The upper level of classification reflects the sign on which the function is formed from the discrepancy between the result of the solution Ur and the result of the observation of the Un. On this basis, the following are distinguished: conventional indicators; threshold; combined.

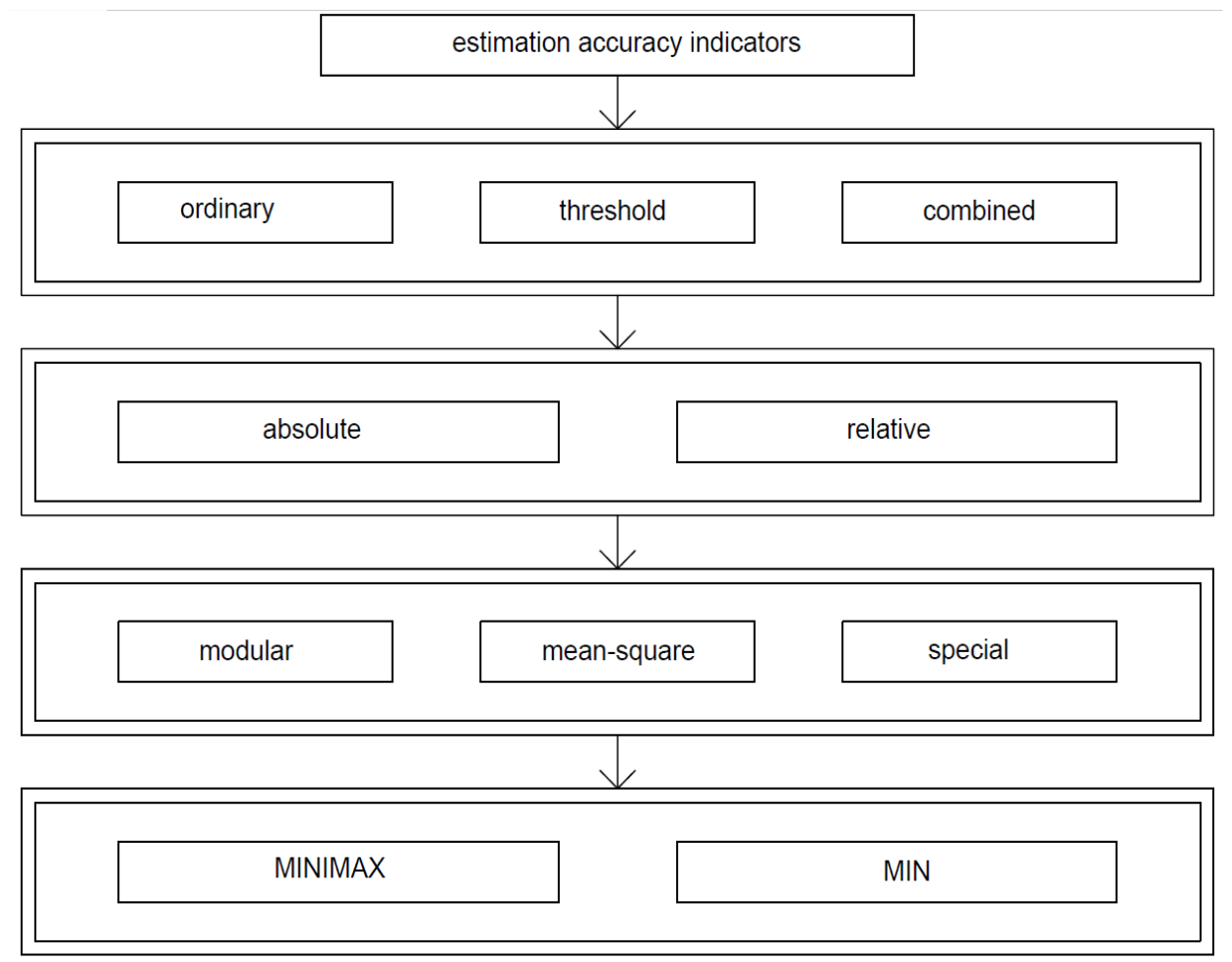

Fig. 3. Classification scheme of accuracy indicators of estimation.

\section{Discussion}

At the beginning of the 20th century, in the book of the engineer F. Taylor "Scientific Management", an unlimited faith in rational methods of cognition was declared. The scientific management was understood as unambiguous, computable, and decomposable into 
elements. F. Taylor believed that the application of strictly scientific principles to the issues of constructing effective organizational structures and processes makes it possible to find the only correct answer to them. The classical scientific management by Taylor is based on four provisions: development of scientifically based knowledge of work; selection and training of employees; combination of knowledge about labor activity with the increased labor capacity of the selected workers; specialization of the types of labor and organizational activities in the form of the distribution of responsibility between managers and employees. Taylor believed that managers should only think, and workers should work. But the requirement to achieve the goal through a strict order, a clearly delineated structure and from the outside of set standards can lead (and usually leads) to the inability of staff and organization managers to adapt flexibly to changing external conditions. The main disadvantage of the Taylor's system is a rigid orientation toward the "economic" model of a person whose main incentive to work is a monetary reward. Up to the present time, the population of Russia has a quite strong content component, unlike the inhabitants of foreign countries, i.e. logical mental activity on the basis of clearly understood ethical norms and the principle of "face to person". F. Taylor considered his system complete and the only possible. However, the reality of business relations by the 1930 s began to refute its principles. All forms and manifestations of thought activity that serve the needs of social activity (theories, functional systems, objects and processes, documents and systems of document circulation, tools and hardware-software complexes for documenting and processing documentation, anthropotechnical methods and technologies, etc.) cannot be considered absolutely "finished" and "only possible". They can be stable only in conditions of a strictly described and unchanged fragment of the process of social relations and the structure of society. As soon as the latter begins to change dynamically, it becomes possible and necessary to change and rearrange all the abovementioned serving needs of a new social formation of forms and manifestations of mental activity.

The emergence and rapid development of technical systems and studying their technical sciences that did not have a place in the structure of the classification of the natural sciences of Aristotle and are a product of mental activity and production activity of man (in contrast to natural sciences, where man studies and establishes the phenomena and laws of nature as a natural habitat) changed the accents and switched attention to the initial stages of choosing and justifying the goals, their usefulness, the conditions of implementation, links with previous prerequisites for processes.

The basic infographic model of the technical field of knowledge, as mentioned above, is the MTE system. The main was the identification of the mechanism of the functioning of the MTE system and the normal functioning of the conditions that violate it. The mechanism of functioning of the MTE includes: the study of the functions of the system, the determination of the relationships of each function with the set of interacting elements of the MTE system, the consideration of the structure of the system not as a relation (interconnection, interaction), but as a defined ordered arrangement of some elements of the MTE system relative to others (relations between relations). Knowledge of the structure and functions of the MTE system is important, but not a sufficient condition for effective solution of problems. It is necessary to relate the goals of the subject-man (the component of the MTE system) to the complex of goals of the MTE system as a single functioning object and to determine how the realization of the goals of the subject-man affects the functioning of the MTE system. In the same way, it should be correlated with the goals of the entire MTE system and the purpose of its other two components - technology and environment. The formation of a consensus of all these goals or rational situations close to such a consensus represents an important practical result of the harmonization of the device of the MTE system.

The MTE systems are explored in three directions: systems science (the theory of MTE systems), system engineering (the practice of the functioning of MTE systems), and system 
analysis (the methodology of the future development of MTE systems). The last of the above named directions needs comment. Initially, the analysis of MTE systems was understood as a complex forecast based on mathematical modeling. The results of the real practice of system engineering led to the conclusion that mathematics is ineffective in the analysis of broad problems with the set of uncertainties that are characteristic for research and development of the technical and system MTE as a whole.

Therefore, they formed the concept of such a system analysis in which emphasis is placed primarily on the development of new dialectical scientific principles for the logical and information-energy research of MTE systems and its components, taking into account their interrelations and contradictory trends. With this approach, not the mathematical methods but the logic of system analysis and the ordering of decision-making procedures are brought to the forefront, understanding a system of system principles and representations under the system approach, in which, apart from the generally accepted, there must be innovative ones. Any principle or conception of the MTE system (generalization) has its own field of applicability in the framework of which they will be right and may turn out to be wrong outside this region.

This is one of the fundamental characteristics of infographics - the positiveness of perception. Many problems arise because they use the model not where it can work (at another position) [10-16, 19-27].

\section{Conclusions}

Our goal - at the level of model experiments of the reconstructed system, to predict the level of comfort of labor activity (and in the long run human activity) as an accentuated component of the "man-technology-environment" (MTE) system, reduce the risk of pathogenic influences and emergency situations in the MTE system not predictable by statistical methods through preliminary diagnostics, monitoring, and adoption of early adequate solutions based on their results. In the long term, the above-mentioned directions of research will allow creating an appropriate array of average regulatory data that can be formalized in the form of officially approved regulatory documents that provide the possibility of their application in the provision of certification services, with the subsequent development of elements of a new model for certification of the system "man-technology-environment". The development of an appropriate regulatory framework using the results of engineering express diagnostics in assessing the quality of industrial and public mobile habitats will ensure the future generation of fundamentally new standards in the provision of certification services for the transport complex. The main advantage of having a certificate is the public recognition of the high quality of the services provided. It is important to note that this approach is universal, i.e. it can be applied to any version of the practical implementation of the "man-technologyenvironment" system in which a person is a priority component. The increase in OTR in optimizing the servicing subsystems of the construction industry is achieved in two ways: by decreasing the level of influence of the factors affecting the failure of the reliability of the functioning of these subsystems (such a reduction is not always possible due to the complexity and often impossibility of influencing these factors) and the creation of subsystems sufficiently reliable functioning in the conditions of action of negative factors. The increase in the MTE of the servicing subsystems of construction production is affected by a decrease in the level of influence of pathogenic factors by combining these two directions, as well as by improving the "operator-workplace" system. Thus, the use of computer information technology CAD tools for mechanization of construction production in the process of monitoring the parameters of the system "man-CMW-activity" makes it possible to increase the comfort and productivity of the worker. 


\section{References}

1. B.A. Lyovin, R.R. Kazaryan, V.O. Chulkov Infographics of anthropotechnical management. Infographic modeling in the mental activity philosophy. 1 (2016)

2. B.A. Lyovin, R.R. Kazaryan, V.O. Chulkov Infographics of anthropotechnical management. Conception of advanced development of anthropotechnical security of functioning and life quality. 2 (2016)

3. B.A. Lyovin, R.R. Kazaryan, V.O. Chulkov Infographics of anthropotechnical management. Anthropotechnical management as a means of provision of activities service. 3 (2016)

4. R.R. Kazaryan, I.A. Bun'kina Sci Rev Regarding some aspects of economic basis of products and services certification system development. 7 (2016)

5. R.R. Kazaryan Sci Rev Regarding some aspects of infographic modeling of road pavement design. 11 (2016)

6. R.R. Kazaryan, I.A. Bun'kina Nat Eng Sci Regarding some aspects of transport system environmental security standardization. 6 (2015)

7. R.R. Kazaryan Nat Eng Sci Regarding some aspects of improvement of construction management efficiency criteria. 11 (2015)

8. R.R. Kazaryan, E.K. Muracheva Nat Eng Sci Regarding some aspects of modeling on improvement the systemic-purposive approach for assessment of efficiency of construction products quality control. 11 (2015)

9. R.R. Kazaryan, I.A. Bun'kina Sci Rev Regarding some aspects of economic basis of development of the systemic-purposive approach for assessment of quality systems elements control. 23 (2015)

10. R.R. Kazaryan Sci Rev Regarding some aspects of use of economic-mathematical methods in transport and road construction. 22 (2015)

11. R.R. Kazaryan, V.O. Chulkov Sci Rev Regarding some aspects of improvement of construction products quality. 24 (2015)

12. V.O. Chulkov, O.N. Kuzina Orgaizational-technological criteria of arrangement of construction renovation varieties. pp 410-426 (2010)

13. V.O. Chulkov, O.N.Kuzina Retrieving - innovative direction in a functional system of construction renovation. // Current problems of housing and municipal services development in cities and population centers: International collection of research papers of the $9^{\text {th }}$ International research and practice conference. pp 439-443 (2010)

14. A.A. Volkov, V.O. Chulkov, G.O. Chulkov, R.R. Kazaryan, O.N. Kyzina Adv Mater Res Qualities of documentation management chain (part 2) 1065-1069, pp 2401-2404 (2015)

15. A.A. Volkov, V.O. Chulkov, G.O. Chulkov, R.R. Kazaryan, O.N. Kyzina Adv Mater Res Qualities of documentation management chain (part 2) 1065-1069, pp 2405-2408 (2015)

16. A.A. Volkov, V.O. Chulkov, G.O. Chulkov, R.R. Kazaryan, O.N. Kyzina Adv Mater Res Qualities of documentation management chain (part 3) 1065-1069, pp 2405-2408 (2015)

17. C. Gini Stats The latest foreign statistical researches. p 448 (2010)

18. A.A. Lapidus, H.L. Saydaev Constr Tech Org Influence of parameters of construction company organizational structure development on the generalized index of environmental load. 1 (2012)

19. R.R. Kazaryan, V.O. Chulkov Internat Coll of Sci Papers About some aspects of the development of the certification system of the transport complex (2012)

20. T.V. Zyuba Industrial safety in transport (2004) 
21. Yu. Hyuvarinen, E.A. Svatkova, I.I. Maksimov, M.F. Felivonchik Trans. And relations between Finland and the Nordic Council of Ministers Principles and tools for improving road safety in human settlements: international experience 1 (2005)

22. Levin B.A., Mamaev E.A. The World of Trans Investment interaction of the subjects of the transportation process 1 (2005)

23. Levin B.A., Ponomarev V.M., Ulyanov V.A. Business Premier Safety in transport. Tasks and solutions. №1-2 (2014)

24. A.N. Lieberman Technogenic safety: the human factor (2006)

25. S.V. Ilinova Engin bulletin of Don Trans Increase of economic efficiency of ecological safety of transport system (2010)

26. K.V.Sudakov, V.O.Chulcov, R.R.Kazaryan, O.S.Glazachev, N.V.Dmitrieva, N.M. Komarov Antropotechnics: Norm in every living thing and artificial beings (2013)

27. K.A. Kirsanov, O.A. Bogomolov Inter Jrnl Naukovedenie Transport revolutions and their consequences (2011) 